\title{
COVID-19 has inspired global healthcare innovation
}

\author{
Adam Palanica $^{1}$ (D) $\cdot$ Yan Fossat $^{1}$
}

Received: 19 May 2020 / Accepted: 18 August 2020 / Published online: 28 August 2020

(C) The Canadian Public Health Association 2020

\begin{abstract}
Under normal circumstances, healthcare innovation is costly and time-consuming. However, the COVID-19 pandemic has produced the silver lining of inspiring healthcare innovation around the world, with collaboration across multiple disciplines all working toward the same goal of saving lives. Healthcare innovation can develop at unprecedented speed when individuals focus on solving real-world problems, and collaborate with cross-functional teams. Anyone can innovate, from anywhere, at any age, and this open-minded perspective allows innovation to occur at its finest when motivated to find solutions toward a welldefined problem.
\end{abstract}

\section{Résumé}

Dans des circonstances normales, l'innovation est coûteuse et prend du temps dans le domaine de la santé. Cependant, la pandémie de COVID-19 a eu comme effet positif de favoriser l'innovation dans les soins de santé partout dans le monde, des acteurs de diverses disciplines œuvrant dans le même but, celui de sauver des vies. L'innovation dans le domaine de la santé peut se développer à une vitesse sans précédent lorsque les gens cherchent à résoudre des problèmes du monde réel et collaborent avec des équipes interfonctionnelles. N'importe qui peut innover, de n'importe où, à n'importe quel âge, une ouverture d'esprit propice à une innovation optimisée lorsqu'il est question de chercher des solutions à un problème bien défini.

Keywords Innovation $\cdot$ Healthcare $\cdot$ COVID-19

Mots-clés Innovation · soins de santé · COVID-19

\section{Innovation in the world of COVID-19}

Innovation. How long does it really take in healthcare? As a benchmark, it takes an average of 12 years to develop a new pharmaceutical drug from bench to market (Kraljevic et al. 2014). Other new technologies and methodologies in healthcare may also take years and several millions or billions of dollars before they are ready for public use. That is under "normal" circumstances. But what if the world is not normal? What if a global pandemic, such as COVID-19, swept through the population, spurring everyone to create innovative healthcare solutions for the current problem? It seems that during these uncertain times, one thing is certain: collaboration from the global population focusing on a real-world

Adam Palanica

apalanica@klick.com

1 Klick Labs, Klick Applied Sciences, Toronto, Ontario, Canada problem is invaluable to developing healthcare innovation at an unprecedented speed. To be clear, other innovation frameworks have been previously developed, but they are either completely theoretical or focus on a narrow field of research. For the first time, the world has now demonstrated bona fide evidence and actionable methods to achieving global healthcare innovation.

In the past few months, the world has seen the pace of healthcare innovation accelerate at a tremendous rate, with the typical timeline of years becoming weeks or days. Solutions to help cope with COVID-19 have been produced at an extraordinary pace, with the added bonus of being relatively inexpensive to implement. A primary example of this comes from a Taiwanese physician who created an "Aerosol box" (Everington n.d.), which is a transparent plastic cube designed to cover patients' heads, with two arm holes for physicians to perform intubations while being protected from the spread of the coronavirus (Canelli et al. n.d.). Importantly, the open-source design has been implemented in clinical practice around the world, within days of first reveal — not years. 
Another example of a practical solution to a real-world problem comes from a 13-year-old who created 3D-printed ear guards for hospital staff to help alleviate the pain caused by wearing face masks for too long (Barnes 2020). Others have developed 3D-printed face shields (Goodyear 2020) and 3Dprinted ventilator valves (Peters 2020) for dealing with COVID-19. Cross-functional teams of physicians, scientists, and web developers have also quickly developed centralized data analytics and visualization hubs to monitor the spread of COVID-19 throughout the world (Johns Hopkins University 2020; U of T News 2020). Innumerable researchers, engineers, clinicians, patients, nurses, and citizen scientists are applying their diverse skills as data analysts, developers, mathematicians, psychologists, designers, and makers toward the same goal of saving lives. Crucially, these solutions are cost-effective, open to the public, and solve fundamental problems, which have practical implications for government and healthcare policy. Although not all ideas are successful, the motivational efforts by these individuals fosters rapid iteration of innovation development.

\section{Keep it simple, silly}

In many circumstances, innovation is fabricated as a shiny object in search of a problem that does not exist. Additionally, the term innovation is usually synonymous with expensive technologies that offer promises without delivering. In recent years, the world of healthcare has been bombarded with buzz words (and buzz acronyms) of artificial intelligence (AI), machine learning (ML), natural language processing (NLP), virtual reality (VR), augmented reality (AR), big data, blockchain, biometric wearables, chatbots, and so on, all being marketed as the "next big thing" for improving health. Sometimes, however, there is very little clinical evidence to support their worth in healthcare. For example, mobile health and fitness applications are ubiquitous in society, yet less than $3 \%$ of wellness apps provide any clinical evidence for their validity (Larsen et al. 2016; Larsen et al. 2019). In some cases, this lack of real-world validity for technology is indeed the catalyst for alternative innovative solutions.

The twenty-first century has seemed to reach a point where flashier and more complex technologies are perceived as more innovative than simpler solutions, even though they may not actually help people. However, innovation is about asking the right questions and focusing on the right problems (Ness 2012). Occam's razor also states that the simplest solutions are more likely to be the correct ones. We have been led astray from this problem-solving principle given the amount of advanced technologies that are around us, and this may lead us to erroneously focus on infeasible solutions when asked to innovate. People can overthink ideas to solve a problem, when an easier solution may be extremely obvious.
Innovation in healthcare does not need to be complicated or expensive (Harris et al. 2018). Regardless of how straightforward the solution may be, a cornerstone of innovation is concentrating on solving the root cause of real problems, albeit within the constraints of accessible resources. Properly defining the problem with input from frontline healthcare workers and patients provides vitally important information to evaluate new ideas; the daily challenges experienced by these individuals should be the focal point of ideation and design. Focusing on simple tactics also allows multiple iterations of testing to fail fast and fail often, and understand what elements work best; innovation is a learning process. During these days of the COVID-19 pandemic, many simple and efficient solutions exist to "flatten the curve", save lives, and decrease the burden on the healthcare system. For example, clearly instructing people to wash their hands, stay home, and increase physical distancing has been instrumental in reducing the spread of the virus, especially through the dissemination of well-designed infographics, news communications, and social media (Chan et al. 2020). Many office employees can work from home and interact with their colleagues through video conferencing. Children can be educated through online school courses. Food delivery services allow people to stay nourished while avoiding crowded stores and restaurants. Online fitness classes allow people to exercise and stay physically active. The hosts of news and talk shows are filming from their own homes to keep audiences happy and entertained. Telemedicine services are more important than ever. Around the world, many people are volunteering their time to deliver goods and services to those in need. These public health efforts are crucial to improving mental and physical health, and their values are held with the utmost respect by the majority of citizens to prevent further disease.

\section{What has changed?}

How could the pace of innovation change so radically during the COVID-19 pandemic? Under non-COVID-19 circumstances, healthcare innovation can be hindered by many barriers, including lack of funding, regulatory approval processes, administrative procedures, ethics considerations, clinical trial testing, and conflicts among stakeholders, such as insurers, medical device companies, pharmaceutical corporations, hospitals, clinicians, and researchers. Priorities can change between the health concerns of patients versus business and marketing developments, which can drag the pace of healthcare improvement. The COVID-19 emergency has elicited a shortening of research and development timelines, predominantly by sidestepping administrative barriers, and focusing on health first and profit second. The innovations that are conceived, built, and utilized are developed from concept to product much faster than under typical conditions. One 
caveat is that these new innovations must still adhere to proper safety and efficacy protocols, and incorporate best practices for patient health and security protections. A reduction of administrative barriers should never be at the sacrifice of human well-being. All innovation initiatives need to be well thought out, examined, and tested before being rushed into practice.

It is also worth noting that increased funding from various grants and lack of executive or business priorities have helped facilitate the increase in COVID-19-related innovation. However, these are not the only reasons. COVID-19 has generated global collaboration across multiple disciplines all working together to aid communities in saving lives. This cross-functionality has been invaluable, with different educational backgrounds, lifestyles, cultures, demographics, skills, and abilities all providing unique perspectives for developing innovation. Cross-functional teams can provide greater breadth and depth of shared information, increase motivation, enhance creativity, improve task coordination, and produce less conflict among individuals (West 2002). None of this requires a need for more money or lenience of bureaucracy, but rather an optimistic outlook on cooperation. The current prosocial climate of altruism and creating open-source datasharing hubs has never been greater. This, in turn, creates an effective and productive method of scaling healthcare innovation to other areas in need of solutions.

Although this is a hopeful perspective on healthcare innovation, there are certainly challenges involved. Innovative ideas should only be reliably implemented after numerous evidence-based research studies and demonstrated safety to patients and providers. This itself can be a difficult barrier to overcome since the gold standard method of double-blind randomized controlled trials may not be entirely feasible during a global pandemic with so many uncertainties of the disease; there is also the ethical debate of whether withholding a potential treatment outweighs the benefit of rigorous scientific experimentation. Additionally, new skills need to be learned by healthcare workers before protocols are fully implemented. Collaboration across multiple disciplines can also be burdensome if physical distancing policies prevent human contact. Ultimately, healthcare innovation is a never-ending learning process that gets refined with continuous clinical evidence. As COVID-19 runs from a sprint to a marathon, scientists and clinicians need to keep sight of the short- and long-term uptake of innovation in policy and practice.

\section{Conclusions}

COVID-19 has negatively affected over 200 countries and millions of people worldwide, but it has also produced the silver lining of inspiring global healthcare innovation.
Along the way, we are learning valuable lessons by datadriven results for future healthcare innovation on a global scale:

- Define problems thoroughly; when possible, gather information from frontline healthcare workers and patients;

- Be open to all new ideas, especially the simple and inexpensive ones; keep it simple, silly;

- Collaborate with others from a wide range of disciplines; cooperation is paramount for public health promotion;

- Acknowledge failure as success, not defeat; innovation involves testing and learning;

- Anyone can innovate, from anywhere, at any age.

Increasing knowledge, shifting mindset, and changing human behaviour are perhaps most pivotal to implementing these principles. Applying these elements to innovation practices will be vital for helping other global dilemmas, such as climate change, excess plastic in oceans, or lack of freshwater supplies. Let us not forget these lessons.

Author contributions A.P. wrote the first draft, with additional drafting by Y.F. Both authors contributed to the story development, as well as reviewing and editing the final content.

\section{Compliance with ethical standards}

Conflict of interest The authors declare that they have no conflict of interest.

\section{References}

Barnes S. Boy scout 3D prints 'ear guards' to help relieve hospital workers' pain caused by face masks. https://mymodernmet.com/ 3d-printed-ear-guards/. Accessed April 29, 2020.

Canelli, R., Connor, C. W., Gonzales, M., Nozari, A., \& Ortega, R. Barrier enclosure during endotracheal intubation [published online April 3, 2020]. The New England Journal of Medicine. https://doi. org/10.1056/NEJMc2007589.

Chan, A. K. M., Nickson, C. P., Rudolph, J. W., Lee, A., \& Joynt, G. M. (2020). Social media for rapid knowledge dissemination: early experience from the COVID-19 pandemic. Anaesthesia.

Everington K. Taiwanese doctor invents device to protect US doctors against coronavirus. Taiwan News https://www.taiwannews.com. tw/en/news/3902435. Accessed April 29, 2020.

Goodyear S. Canadian doctor who works in Gaza makes 3D-printed face shields for COVID-19. https://www.cbc.ca/radio/asithappens/as-ithappens-thursday-edition-1.5502954/canadian-doctor-who-worksin-gaza-makes-3d-printed-face-shields-for-covid-19-1.5502964. Accessed April 29, 2020.

Harris, M., Bhatti, Y., Prime, M., del Castillo, J., \& Parston, G. (2018). Low-cost innovation in healthcare: what you find depends on where you look. Journal of the Royal Society of Medicine, 111(2), 47-50.

Johns Hopkins University. COVID-19 Dashboard. https://coronavirus. jhu.edu/map.html. Accessed April 29, 2020.

Kraljevic, S., Stambrook, P. J., \& Pavelic, K. (2014). Accelerating drug discovery. EMBO Reports, 5(9), 837-842. 
Larsen, M. E., Nicholas, J., \& Christensen, H. (2016). Quantifying app store dynamics: longitudinal tracking of mental health apps. JMIR mHealth and uHealth, 4(3), e96.

Larsen, M. E., Huckvale, K., Nicholas, J., et al. (2019). Using science to sell apps: evaluation of mental health app store quality claims. NPJ Digital Medicine, 2, 18.

Ness, R. B. (2012). Innovation generation: How to produce creative and useful scientific ideas. Oxford: Oxford University Press.

Peters J. Volunteers produce 3D-printed valves for life-saving coronavirus treatments. https://www.theverge.com/2020/3/17/21184308/ coronavirus-italy-medical-3d-print-valves-treatments. Accessed April 29, 2020
$\mathrm{U}$ of $\mathrm{T}$ News. \#HowsMyFlattening? Group led by $\mathrm{U}$ of $\mathrm{T}$ community members creates one-stop shop for COVID-19 data. https:/www. utoronto.ca/news/howsmyflattening-group-led-u-t-communitymembers-creates-one-stop-shop-covid-19-data. Accessed April 29, 2020.

West, M. A. (2002). Sparkling fountains or stagnant ponds: an integrative model of creativity and innovation implementation in work groups. Applied Psychology, 51, 355-387.

Publisher's note Springer Nature remains neutral with regard to jurisdictional claims in published maps and institutional affiliations. 